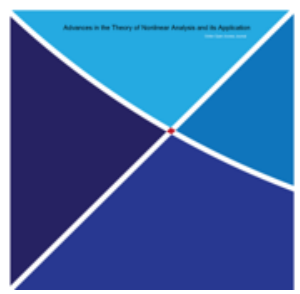

\title{
Blow up and asymptotic behavior for a system of viscoelastic wave equations of Kirchhoff type with a delay term
}

Fatima Zohra Mahdia, Mohamed Ferhat ${ }^{a}$, Ali Hakem $^{a}$

a Laboratory ACEDP, Djillali Liabes University, 22000 Sidi Bel Abbes, Algeria.

\section{Abstract}

The focus of the current paper is to investigate the initial boundary value problem for a system of viscoelastic wave equations of Kirchhoff type with a delay term in a bounded domain. At first, the energy decay rate is proved by Nakao's technique and expressed polynomially and exponentially depending on the parameter $m$. However and in the unstable set, for certain initial data, the blow up of solutions is obtained.

Keywords: Global existence, Energy decay, Blow up of solutions, Nonlinear damping.

2010 MSC: 35L60, 35K55, 26A33; 35B44, 35B33.

\section{Introduction}

In this paper, we are concerned with the following problem:

$$
\begin{gathered}
u_{t t}-M\left(\|\nabla u\|_{2}^{2}\right) \Delta u+\int_{0}^{t} g(t-s) \Delta u(s) d s+\mu_{1}\left|u_{t}(x, t)\right|^{m-1} u_{t}(x, t) \\
+\mu_{2}\left|u_{t}(x, t-\tau)\right|^{m-1} u_{t}(x, t-\tau)=|u|^{p-1} u, \\
u_{t}(x, t-\tau)=f_{0}(x, t-\tau), x \in \Omega, t \in(0, \tau), \\
u(x, 0)=u_{0}(x), u_{t}(x, 0)=u_{1}(x), x \in \Omega,
\end{gathered}
$$

Email addresses: mahdifatimazohra@yahoo.fr (Fatima Zohra Mahdi), ferhat22@hotmail.fr (Mohamed Ferhat), hakemali@yahoo.com (Ali Hakem) 


$$
u(x, t)=0, x \in \partial \Omega, t \geq 0 .
$$

Here $\Omega$ is a bounded domain in $\mathbb{R}^{n}, n \in \mathbb{N}^{*}$, with a smooth boundary $\partial \Omega . \tau>0$ is a time delay term and $\mu_{1}, \mu_{2}$ are positive real numbers and $p>1$. The initial datum $\left(u_{0}, u_{1}, f_{0}\right)$ belong to a suitable spaces.

To motivate our work, let us recall some results regarding wave equations of Kirchhoff type.

This type of problem without delay (i.e., $\mu_{2}=0$ ) has been considered by many authors during the past decades and many results have been obtained (see [9], [18], [22, [24] ) and the references therein.

The problem (1.1)-(1.4) without the viscoelastic term and delay (i.e., $g=0, \mu_{2}=0$ ) has been extensively studied and decay and blow-up have been established. For example, the following equation

$$
u_{t t}-M\left(\|\nabla u\|_{2}^{2}\right) \Delta u+g\left(u_{t}\right)=f(u),
$$

has been considered by Matsuyama and Ikehata in [18], for $g\left(u_{t}\right)=\delta\left|u_{t}\right|^{p} u_{t}$ and $f(u)=\xi|u|^{p} u$. The authors proved existence of the global solutions by using Faedo-Galerkin's method and the decay of energy based on the method of Nakao [20]. Later, Ono [21] investigated equation (1.5) for $M(s)=b s, f(u)=\xi|u|^{p} u$ and $g\left(u_{t}\right)=-\Delta u_{t}$. They showed that the solutions blow up in finite time with $E(0) \leq 0$. For $M(s)=a+b s$ and $g\left(u_{t}\right)=u_{t}$, this model was considered by the same author in [22]. By applying the potential well method, he obtained the blow-up properties with positive initial energy $E(0)$.

Recently, Zeng et al. 24] have studied equation (1.5) for the case $g\left(u_{t}\right)=u_{t}$ with initial condition and zero Dirichlet boundary condition. By using the concavity argument, they proved that the solutions to equation 1.5 blows up in finite time with arbitrarily high energy.

When $g \neq 0$ and $M$ is not a constant function, problems related to 1.5 have been treated by many authors. Wu and Tsai [23] considered the global existence, asymptotic behavior and blow-up properties for the following equation

$$
u_{t t}-M\left(\|\nabla u\|_{2}^{2}\right) \Delta u+\int_{0}^{t} g(t-s) \Delta u(s) d s-\Delta u_{t}=f(u),
$$

where $(x, t) \in \Omega \times(0, \infty)$ and with the same initial and boundary conditions as that of problem (1.1)(1.4). To obtain the decay result, they assumed that the nonnegative kernel $g^{\prime}(t) \leq-r g(t) \forall t \geq 0$ for some $r>0$. Later, $\mathrm{Wu}$ [25], extended the result of [23] under a weaker condition on $g$ (i.e $g^{\prime}(s) \leq 0$ for $t \geq 0$ ).

In the present paper, we analyze the influence of the viscoelastic, damping and delay terms on the solutions to (1.1)-(1.4). Under suitable assumptions on the function $g$, the initial data and the parameters in the equations, we establish several results concerning asymptotic behavior and finite blow-up of solutions to 1.1)-1.4 for both negative and positive initial energy.

The paper is organized as follows. In Section 2, we present the preliminaries and some lemmas. In Section 3, the decay property is discussed. Finally, in Section 4, the blow-up results of (1.1)-(1.4) are obtained on different cases of the sign of the initial energy $E(0)$.

\section{Preliminary Results}

In this section, we present some material for the proof of our result. We assume

$\left(A_{0}\right) M(s)$ is a nonnegative $C^{1}$ function for $s \geq 0$ satisfying

$$
M(s)=m_{0}+\alpha s^{\gamma}, m_{0}>0, \quad \alpha \geq 0 \text { and } \gamma \geq 0 .
$$

$\left(A_{1}\right) g: \mathbb{R}^{+} \rightarrow \mathbb{R}^{+}$is a bounded $C^{1}$ function satisfying

$$
g(s) \geq 0, m_{0}-\int_{0}^{\infty} g(s) d s=l>0, g^{\prime}(t) \leq-r g(t), \forall t \geq 0, \quad \text { and } g^{\prime}(s) \leq 0 .
$$


$\left(A_{2}\right)$ We assume that

$$
\beta \bar{M}(s)-\left[M(s)+\eta \int_{0}^{t} g(\nu) d \nu\right] s \geq m_{1} s, \forall s \geq 0
$$

where $\bar{M}(s)=\int_{0}^{s} M(\nu) d \nu, m_{1}>0, \beta$ and $\eta$ are positive constants that will be specified later.

Lemma 2.1. (Sobolev-Poincaré inequality) Let $2 \leq m \leq \frac{2 n}{n-2}$. The inequality

$$
\|u\|_{m} \leq c_{s}\|\nabla u\|_{2} \quad \text { for } \quad u \in H_{0}^{1}(\Omega)
$$

holds with some positive constant $c_{s}$.

Lemma 2.2. ([14]) Let $\phi(t)$ be a nonincreasing and nonnegative function on $[0, T], T>1$, such that

$$
\phi(t)^{1+r} \leq \omega_{0}(\phi(t)-\phi(t+1)), \quad \text { on }[0, T],
$$

where $\omega_{0}>1$ and $r \geq 0$. Then we have, for all $t \in[0, T]$

(i) if $r=0$, then

$$
\phi(t) \leq \phi(0) e^{-\omega_{1}[t-1]^{+}},
$$

(ii) if $r>0$, then

$$
\phi(t) \leq\left(\phi(0)^{-r}+\omega_{0}^{-1} r[t-1]^{+}\right)^{\frac{-1}{r}},
$$

where $\omega_{1}=\ln \left(\frac{\omega_{0}}{\omega_{0}-1}\right)$ and $[t-1]^{+}=\max (t-1,0)$.

Lemma 2.3. ([26]) For any $g \in C^{1}$ and $\varphi \in H^{1}(0, T)$, we have

$$
\left.\int_{0}^{t} \int_{\Omega} g(t-s) \varphi \varphi_{t} d x d s=-\frac{1}{2} \frac{d}{d t}(g o \varphi)(t)+\int_{0}^{t} g(s) d s\|\varphi\|_{2}^{2}\right)-g(t)\|\varphi\|_{2}^{2}+\left(g^{\prime} \circ \varphi\right)(t)
$$

where

$$
(g \circ \varphi)(t)=\int_{0}^{t} g(t-s) \int_{\Omega}|\varphi(s)-\varphi(t)|^{2} d x d s .
$$

Lemma 2.4. ([26]). For $u \in H_{0}^{1}(\Omega)$, we have

$$
\int_{\Omega}\left(\int_{0}^{t} g(t-s)(u(t)-u(s)) d s\right)^{2} d x \leq(1-l) c_{s}^{2}(g o \nabla u)(t)
$$

where $c_{s}^{2}$ is the Poincaré constant and $l$ is given in $\left(A_{1}\right)$. 


\section{Global existence and asymptotic behavior}

In order to prove the global existence result, we introduce the new variable $z$ as in [12],

$$
z(x, k, t)=u_{t}(x, t-\tau k), x \in \Omega, k \in(0,1),
$$

which implies that

$$
\tau z_{t}(x, k, t)+z_{k}(x, k, t)=0, \quad \text { in } \quad \Omega \times(0,1) \times(0, \infty) .
$$

Therefore, problem 1.1 -1.4 can be transformed as follows

$$
\left\{\begin{array}{l}
u_{t t}-M\left(\|\nabla u\|_{2}^{2}\right) \Delta u+\int_{0}^{t} g(t-s) \Delta u(s) d s \\
+\mu_{1}\left|u_{t}(x, t)\right|^{m-1} u_{t}(x, t)+\mu_{2}|z(x, 1, t)|^{m-1} z(x, 1, t) \\
=|u|^{p-1} u, \quad \text { in } \Omega \times(0, \infty), \\
\tau z_{t}(x, k, t)+z_{k}(x, k, t)=0, \quad \text { in } \Omega \times(0,1) \times(0, \infty) \\
z(x, k, 0)=f_{0}(x,-\tau k), x \in \Omega, \\
u(x, 0)=u_{0}(x), u_{t}(x, 0)=u_{1}(x), x \in \Omega \\
u(x, t)=0, x \in \partial \Omega, t \geq 0 .
\end{array}\right.
$$

For any regular solution of (3.1), we define the energy as

$$
\begin{aligned}
& E(t)=\frac{1}{2}\left\|u_{t}\right\|_{2}^{2}+\frac{1}{2}(g o \nabla u)(t)+\frac{1}{2}\left(m_{0}-\int_{0}^{t} g(s) d s\right)\|\nabla u\|_{2}^{2} \\
& -\frac{1}{p+1}\|u\|_{p+1}^{p+1}+\frac{\alpha}{2(\gamma+1)}\left(\|\nabla u\|_{2}^{2}\right)^{\gamma+1}+\frac{\zeta}{2} \int_{\Omega} \int_{0}^{1} z^{m+1}(x, k, s) d k d x
\end{aligned}
$$

such that $\zeta$

$$
\tau \frac{\mu_{2} m}{m+1}<\zeta<\tau \frac{\mu_{1}(m+1)-\mu_{2}}{m+1} .
$$

Lemma 3.1. Let $(u, z)$ be the solution of (3.1), then the energy satisfies

$$
\begin{aligned}
& E^{\prime}(t)=\frac{1}{2}\left(g^{\prime} o \nabla u\right)(t)-\left(\mu_{1}-\frac{\zeta}{2 \tau}-\frac{\mu_{2}}{m+1}\right)\left\|u_{t}(t)\right\|_{m+1}^{m+1} \\
& -\left(\frac{\zeta}{\tau}-\frac{\mu_{2} m}{m+1}\right) \int_{\Omega} z^{m+1}(x, 1, t) d x-\frac{1}{2} g(t)\|\nabla u(t)\|_{2}^{2} \leq 0 .
\end{aligned}
$$

Proof. Multiplying the first equation in (3.1) by $u_{t}$, integrating over $\Omega$ and using integration by parts, we get

$$
\begin{aligned}
& \frac{d}{d t}\left[\frac{1}{2}\left\|u_{t}(t)\right\|_{2}^{2}+\frac{1}{2} m_{0}\|\nabla u(t)\|_{2}^{2}+\frac{\alpha}{2(\gamma+1)}\left(\|\nabla u\|_{2}^{2}\right)^{\gamma+1}\right. \\
& \left.-\frac{1}{p+1}\|u\|_{p+1}^{p+1}\right]+\mu_{1}\left\|u_{t}(t)\right\|_{m+1}^{m+1} \\
& +\int_{\Omega} \mu_{2}|z(x, 1, t)|^{m-1} z(x, 1, t) u_{t}(x, t) d x \\
& -\int_{0}^{t} g(t-s) \int_{\Omega} \nabla u(s) \nabla u_{t}(t) d x d s=0 .
\end{aligned}
$$


Using Lemma 2.3 on the last term of the left hand side of $(3.5)$, we find

$$
\begin{aligned}
& \frac{d}{d t}\left[\frac{1}{2}\left\|u_{t}(t)\right\|_{2}^{2}+\frac{1}{2}\left(m_{0}-\int_{0}^{t} g(s) d s\right)\|\nabla u(t)\|_{2}^{2}\right. \\
& \left.+\frac{1}{2}(g o \nabla u)(t)+\frac{\alpha}{2(\gamma+1)}\left(\|\nabla u\|_{2}^{2}\right)^{\gamma+1}-\frac{1}{p+1}\|u\|_{p+1}^{p+1}\right] \\
& +\mu_{1}\left\|u_{t}(t)\right\|_{m+1}^{m+1}+\mu_{2} \int_{\Omega}|z(x, 1, t)|^{m-1} z(x, 1, t) u_{t}(x, t) d x \\
& +\frac{1}{2} g(t)\|\nabla u(t)\|_{2}^{2}-\frac{1}{2}\left(g^{\prime} o \nabla u\right)(t)=0 .
\end{aligned}
$$

Integrating (3.6) over $(0, t)$ we arrive at

$$
\begin{aligned}
& {\left[\frac{1}{2}\left\|u_{t}(t)\right\|_{2}^{2}+\frac{1}{2}\left(m_{0}-\int_{0}^{t} g(s) d s\right)\|\nabla u(t)\|_{2}^{2}+\frac{1}{2}(g o \nabla u)(t)\right.} \\
& \left.+\frac{\alpha}{2(\gamma+1)}\left(\|\nabla u\|_{2}^{2}\right)^{\gamma+1}-\frac{1}{p+1}\|u\|_{p+1}^{p+1}\right]+\int_{0}^{t} \mu_{1}\left\|u_{s}(s)\right\|_{m+1}^{m+1} d s \\
& +\mu_{2} \int_{0}^{t} \int_{\Omega}|z(x, 1, s)|^{m-1} z(x, 1, s) u_{s}(x, s) d s d x \\
& -\frac{1}{2} \int_{0}^{t} g(s)\left(g^{\prime} o \nabla u\right)(s) d s+\frac{1}{2} \int_{0}^{t} g(s)\|\nabla u(s)\|_{2}^{2} d s \\
& =\frac{1}{2}\left\|u^{1}\right\|_{2}^{2}+\frac{1}{2}\left\|\nabla u^{0}\right\|_{2}^{2} .
\end{aligned}
$$

Multiplying the second equation in $(3.1)$ by $\zeta|z|^{m-1} z$ and integrating the result over $\Omega \times(0,1)$ we obtain

$$
\begin{aligned}
& \zeta \frac{d}{d t} \int_{\Omega} \int_{0}^{1}|z(x, k, t)|^{m-1} z(x, k, t) d k d x= \\
& -\frac{\zeta}{\tau(m+1)} \int_{\Omega} \int_{0}^{1} \frac{\partial}{\partial k}|z(x, k, t)|^{m+1} d k d x \\
& =-\frac{\zeta}{\tau(m+1)} \int_{\Omega}\left[|z(x, 1, t)|^{m+1}-|z(x, 0, t)|^{m+1}\right] d x \\
& =-\frac{\zeta}{\tau} \int_{\Omega}|z(x, 1, t)|^{m+1} d x+\frac{\zeta}{\tau}\left\|u_{t}(t)\right\|_{m+1}^{m+1} .
\end{aligned}
$$

Combining (3.7) and (3.8) together, we get

$$
\begin{aligned}
& E(t)+\left(\mu_{1}-\frac{\zeta}{\tau}\right) \int_{0}^{t}\left\|u_{s}(s)\right\|_{m+1}^{m+1} d s+\frac{\zeta}{\tau} \int_{0}^{t} \int_{\Omega}|z(x, 1, s)|^{m+1} d s d x \\
& -\mu_{2} \int_{0}^{t} \int_{\Omega}|z(x, 1, s)|^{m-1} z(x, 1, s) u_{s}(x, s) d s d x \\
& +\frac{1}{2} \int_{0}^{t} g(s)\|\nabla u(s)\|_{2}^{2} d s-\frac{1}{2} \int_{0}^{t}\left(g^{\prime} o \nabla u\right)(s) d s=E(0) .
\end{aligned}
$$

Making use of the Young's inequality on the fourth term of the left hand side of (3.9), we deduce that

$$
\begin{aligned}
& E(t)+\left(\mu_{1}-\frac{\zeta}{\tau}-\frac{\mu_{2}}{m+1}\right) \int_{0}^{t}\left\|u_{s}(s)\right\|_{m+1}^{m+1} d s \\
& -\left(\frac{\zeta}{\tau}-\frac{\mu_{2} m}{m+1}\right) \int_{\Omega} \int_{0}^{t} z^{m+1}(x, 1, s) d s d x \\
& +\frac{1}{2} \int_{0}^{t} g(s)\|\nabla u(s)\|_{2}^{2} d s-\frac{1}{2} \int_{0}^{t}\left(g^{\prime} o \nabla u\right)(s) d s=E(0) .
\end{aligned}
$$


After deriving (3.10), we get the desired result. This completes the proof.

Remark 3.2. Due to the condition (3.3) we have

$$
c_{1}=\left(\mu_{1}-\frac{\zeta}{\tau}-\frac{\mu_{2}}{m+1}\right)>0, \quad c_{2}=\left(\frac{\zeta}{\tau}-\frac{\mu_{2} m}{m+1}\right)>0 .
$$

Remark 3.3. The conditions $\left(A_{2}\right)$ is used to derive the blow up of solution to problem (3.1).

We show that when $m \geq p$ the solution of the problem (3.1) is global if

$$
\frac{n}{n-2}>m \geq p>1, n \geq 3 ;(1<p<m, n \leq 2) .
$$

Now, we center our attention on the global existence of the solutions to the problem (3.1). In order to do so, as in [6], we define

$$
\begin{aligned}
I_{1}(t) & =\left(m_{0}-\int_{0}^{t} g(s) d s\right)\|\nabla u\|_{2}^{2}+(g o \nabla u)(t)-\|u\|_{p+1}^{p+1} \\
& +\zeta \int_{\Omega} \int_{0}^{1} z^{m+1}(x, k, s) d k d x \\
I_{2}(t) & =\left(m_{0}-\int_{0}^{t} g(s) d s\right)\|\nabla u\|_{2}^{2}+(g o \nabla u)(t)-\|u\|_{p+1}^{p+1} \\
& +\alpha\left(\|\nabla u\|_{2}^{2}\right)^{\gamma+1}+\zeta \int_{\Omega} \int_{0}^{1} z^{m+1}(x, k, s) d k d x,
\end{aligned}
$$

and

$$
\begin{aligned}
J(t) & =\frac{1}{2}(g o \nabla u)(t)+\frac{1}{2}\left(m_{0}-\int_{0}^{t} g(s) d s\right)\|\nabla u\|_{2}^{2}-\frac{1}{p}\|u\|_{p}^{p} \\
& +\frac{\alpha}{2(\gamma+1)}\left(\|\nabla u\|_{2}^{2}\right)^{\gamma+1}+\frac{\zeta}{m+1} \int_{\Omega} \int_{0}^{1} z^{m+1}(x, k, s) d k d x .
\end{aligned}
$$

We observe that

$$
E(t)=J(t)+\frac{1}{2}\left\|u_{t}\right\|_{2}^{2}
$$

and

$$
I_{2}(t)=I_{1}(t)+\alpha\left(\|\nabla u\|_{2}^{2}\right)^{\gamma+1} .
$$

Lemma 3.4. Suppose that $\left(A_{0}\right)-\left(A_{1}\right)$ and (3.11) hold. Let $(u, z)$ be the solution of the problem (3.1). Assume further that $I_{1}(0)>0$ and

$$
\alpha=\left(\frac{2(p+1)}{p-1} E(0)\right)^{\frac{p-1}{2}}<1 .
$$

Then $I_{1}(t)>0$ for all $t \geq 0$.

Proof. Since $I(0)>0$, then there exists (by continuity of $u(t)) T^{*}<T$ such that

$$
I(t) \geq 0, \quad \text { for all } t \in\left[0, T^{*}\right] .
$$


From (3.14) and 3.15) we get easily

$$
\begin{aligned}
J(t) & \geq \frac{p-1}{2(p+1)}\left[\left(m_{0}-\int_{0}^{t} g(s) d s\right)\|\nabla u\|_{2}^{2}+(g o \nabla u)(t)\right] \\
& +\frac{p-1}{2(p+1)}\left[\zeta \int_{0}^{1} \int_{\Omega} z^{m+1}(x, k, t) d k d x\right]+\frac{1}{p+1} I_{1}(t) \\
& \geq \frac{p-1}{2(p+1)}\left[\left(m_{0}-\int_{0}^{t} g(s) d s\right)\|\nabla u\|_{2}^{2}+(g o \nabla u)(t)\right. \\
& \left.+\zeta \int_{0}^{1} \int_{\Omega} z^{m+1}(x, k, t) d k d x\right] \geq \frac{p-1}{2(p+1)} l\|\nabla u\|_{2}^{2} .
\end{aligned}
$$

Thus by (3.10) and (3.19) we deduce $\forall t \in\left[0, T^{*}\right]$

$$
l\|\nabla u\|_{2}^{2} \leq\left(1-\int_{0}^{t} g(s) d s\right)\|\nabla u\|_{2}^{2} \leq \frac{2(p+1)}{(p-1)} E(t) \leq \frac{2(p+1)}{(p-1)} E(0) .
$$

Exploiting Lemma 2.1 and formula (3.17), we obtain

$$
\begin{aligned}
\|u\|_{p+1}^{p+1} & \leq c_{s}^{p+1}\|\nabla u\|_{2}^{p+1} \leq \frac{c_{s}^{p+1}}{l}\|\nabla u\|_{2}^{p-1}\|\nabla u\|_{2}^{2} \\
& \leq \frac{c_{s}^{p+1}}{l}\left(\frac{2(p+1)}{(p-1) l} E(0)\right)^{\frac{p-1}{2}} l\|\nabla u\|_{2}^{2} \\
& =\alpha l\|\nabla u\|_{2}^{2}<\left(m_{0}-\int_{0}^{t} g(s) d s\right)\|\nabla u\|_{2}^{2}, \forall t \in\left[0, T^{*}\right] .
\end{aligned}
$$

Whereupon

$$
I_{1}(t)=\left(m_{0}-\int_{0}^{t} g(s) d s\right)\|\nabla u\|_{2}^{2}+(g o \nabla u)(t)-\|u\|_{p+1}^{p+1}+\zeta \int_{0}^{1} \int_{\Omega} z^{m+1}(x, k, t) d k d x>0, \forall t \in\left[0, T^{*}\right] .
$$

Repeating this procedure and using the fact that

$$
\lim _{t \rightarrow T^{*}} \frac{c_{s}^{p+1}}{l}\left(\frac{2(p+1)}{2 l(p-1)} E(u(t))\right)^{\frac{p-1}{2}} \leq \alpha<1 .
$$

We can take $T^{*}=T$. This completes the proof.

Theorem 3.5. Assume that the assumptions $\left(A_{0}\right)-\left(A_{1}\right)$ are fulfilled. Let $u_{0} \in H_{0}^{1}(\Omega) \cap H^{2}(\Omega), u_{1} \in H_{0}^{1}(\Omega)$ and $f_{0} \in L^{2}(\Omega \times(0,1))$ be given. Suppose that (3.11) and $\mu_{2}<\mu_{1}$ hold. Then the solution of the problem (3.1) is global and bounded in time. Furthermore, if

$$
m_{0}<\frac{2 \theta l+l^{2}+4 l}{4+l}
$$

then we have the following decay estimates:

(i) if $m=1$

$$
\phi(t) \leq \phi(0) e^{-\omega_{1}[t-1]^{+}},
$$

where $\omega_{1}=\ln \left(\frac{\omega_{0}}{\omega_{0}-1}\right)$, and $[t-1]^{+}=\max (t-1,0)$,

(ii) if $m>1$

$$
\phi(t) \leq\left(\phi(0)^{-m}+\tau_{2} m[t-1]^{+}\right)^{\frac{-1}{m}} \text { for } t \geq 0,
$$

with $\tau_{2}=c_{14}^{-1}(E(0))$. 
Proof. First, we prove that $T=\infty$. It is sufficient to show that $l\|\nabla u\|_{2}^{2}$ is bounded independently of $t$. We have from (3.14) and 3.15

$$
E(0) \geq E(t)=\frac{1}{2}\left\|u_{t}\right\|_{2}^{2}+J(t) \geq \frac{1}{2}\left\|u_{t}\right\|_{2}^{2}+\left(\frac{p-1}{2(p+1)}\right)\left(l\|\nabla u\|_{2}^{2} \geq\left(l\|\nabla u\|_{2}^{2}\right) .\right.
$$

Therefore

$$
\left(l\|\nabla u\|_{2}^{2}\right) \leq \xi E(0),
$$

where $\xi$ is a positive constant which depends only on $p$, thus we obtain the global existence result. This ends the proof.

From now and on, we focus our attention to the decay rate of the solutions to problem (3.1). In order to do so, we will derive the decay rate of the energy function for problem (3.1) by Nakao's method [14]. For this aim, we have to show that the energy function defined by (3.15) satisfies the hypotheses of Lemma 2.2. By integrating (3.4) over $[t, t+1]$, we have

$$
E(t)-E(t+1)=[D(t)]^{m+1},
$$

where

$$
\begin{aligned}
{[D(t)]^{m+1} } & =c_{1} \int_{t}^{t+1}\left\|u_{t}\right\|_{m+1}^{m+1} d s+c_{2} \int_{t}^{t+1} \int_{\Omega} z^{m+1}(x, 1, s) d x d s \\
& +\int_{t}^{t+1} \frac{1}{2}\left(g^{\prime} o \nabla u\right)(s) d s-\int_{t}^{t+1} \frac{1}{2} g(s)\|\nabla u(s)\| d s .
\end{aligned}
$$

By virtue of (3.24) and Holder's inequality, we observe that

$$
\int_{t}^{t+1}\left|u_{t}\right|^{2} d x d t+\int_{t}^{t+1} \int_{\Omega}|z(x, 1, s)|^{2} d x d s \leq c(\Omega)[D(t)]^{2},
$$

where $c(\Omega)=\operatorname{vol}(\Omega)$. Applying the mean value, there exist $t_{1} \in\left[t, t+\frac{1}{4}\right]$ and $t_{2} \in\left[t+\frac{3}{4}, t+1\right]$ such that

$$
\| u_{t}\left(t_{i}\left\|_{2}^{2}+\right\| z\left(x, 1, t_{i}\right) \|_{2}^{2} \leq c(\Omega) D(t)^{2}, i=1,2 .\right.
$$

Multiplying the first equation in (3.1) by $u$ and integrating over $\Omega \times\left[t_{1}, t_{2}\right]$. Using integration by parts, Holder's inequality, adding and subtracting the following term $\int_{\Omega} \zeta z^{m+1}(x, k, t) d k d x$, we obtain

$$
\begin{aligned}
& \int_{t_{1}}^{t_{2}} I_{2}(t) d t \leq \sum_{i=1}^{2}\left\|u_{t}\left(t_{i}\right)\right\|_{2}\left\|u\left(t_{i}\right)\right\|_{2}+\int_{t_{1}}^{t_{2}}\left\|u_{t}\right\|_{2}^{2} d t \\
& -\mu_{1} \int_{t_{1}}^{t_{2}} \int_{\Omega}\left|u_{t}\right|^{m-1}\left|u_{t}\right| u d x d t+\int_{t_{1}}^{t_{2}}(g o \nabla u)(t) d t \\
& +\int_{t_{1}}^{t_{2}} \int_{\Omega} \int_{0}^{t} g(t-s) \nabla u(t)(\nabla u(s)-\nabla u(t)) d s d x d t \\
& +\int_{t_{1}}^{t_{2}} \int_{0}^{1} \zeta \int_{\Omega} z^{m+1}(x, k, t) d k d x d t \\
& -\mu_{2} \int_{\Omega} \int_{t_{1}}^{t_{2}}|z(x, 1, t)|^{m-1} z(x, 1, t) u d t d x .
\end{aligned}
$$


Since

$$
\begin{aligned}
& \int_{\Omega} \int_{0}^{t} g(t-s) \nabla u(t)(\nabla u(s)-\nabla u(t)) d s d x \\
& =\frac{1}{2}\left[\int_{0}^{t} g(t-s)\left(\|\nabla u(s)\|_{2}^{2}+\|\nabla u(t)\|_{2}^{2}\right) d s\right. \\
& \left.-\int_{0}^{t} g(t-s)\left(\|\nabla u(s)\|_{2}^{2}+\|\nabla u(t)\|_{2}^{2}\right) d s\right] \\
& -\int_{\Omega} \int_{0}^{t} g(s)|\nabla u(t)|^{2} d s d x=-\frac{1}{2} \int_{\Omega} \int_{0}^{t} g(s)|\nabla u(s)|^{2} d s d x \\
& +\frac{1}{2} \int_{0}^{t} g(t-s) d s\|\nabla u(s)\|_{2}^{2} d s-\frac{1}{2}(g o \nabla u)(t) .
\end{aligned}
$$

Then (3.27) takes the form

$$
\begin{aligned}
& \int_{t_{1}}^{t_{2}} I_{2}(t) d t \leq \sum_{i=1}^{2}\left\|u_{t}\left(t_{i}\right)\right\|_{2}\left\|u\left(t_{i}\right)\right\|_{2}+\int_{t_{1}}^{t_{2}}\left\|u_{t}\right\|_{2}^{2} d t \\
& +\mu_{1} \int_{t_{1}}^{t_{2}} \int_{\Omega}\left|u_{t}\right|^{m-1}\left|u_{t}\right| u d x d t+\frac{1}{2} \int_{t_{1}}^{t_{2}}(g o \nabla u)(t) d t \\
& -\frac{1}{2} \int_{\Omega} \int_{0}^{t} g(s)|\nabla u(s)|^{2} d s d x+\frac{1}{2} \int_{0}^{t} g(t-s) d s\|\nabla u(s)\|_{2}^{2} d s \\
& +\int_{t_{1}}^{t_{2}} \int_{0}^{1} \zeta \int_{\Omega} z^{m+1}(x, k, t) d k d x d t \\
& -\mu_{2} \int_{\Omega} \int_{t_{1}}^{t_{2}}|z(x, 1, t)|^{m-1} z(x, 1, t) u d t d x .
\end{aligned}
$$

Now we will estimate the right hand side of $(3.29)$. First by 3.20$),(3.26)$ and Lemma 2.1, we have

$$
\begin{aligned}
\left\|u_{t}\left(t_{i}\right)\right\|_{2}\left\|u\left(t_{i}\right)\right\|_{2} & \leq c_{s} c(\Omega)^{\frac{1}{2}} D(t) \sup _{t_{1} \leq s \leq t_{2}}\|\nabla u(s)\|_{2} \\
& \leq D(t) c_{s} c(\Omega)^{\frac{1}{2}}\left(\frac{2(p+1)}{p-1}\right)^{\frac{1}{2}} \sup _{t_{1} \leq s \leq t_{2}}(E(s))^{\frac{1}{2}} \\
& \leq D(t) c_{s} c(\Omega)^{\frac{1}{2}}\left(\frac{2(p+1)}{p-1}\right)^{\frac{1}{2}}(E(t))^{\frac{1}{2}} .
\end{aligned}
$$

Employing Young's inequality for convolution $\|\varphi * \phi\| \leq\|\varphi\|\|\phi\|$ and noting that

$$
l\|\nabla u(t)\|_{2}^{2} \leq \frac{1}{\theta} I_{2}(t)
$$

then we have

$$
\begin{aligned}
& \int_{0}^{t} \int_{t_{1}}^{t_{2}} g(t-s)\|\nabla u(s)\|_{2}^{2} d s d t \leq \int_{t_{1}}^{t_{2}} g(t) d t \int_{t_{1}}^{t_{2}}\|\nabla u(t)\|_{2}^{2} d t \\
& \leq\left(m_{0}-l\right) \int_{t_{1}}^{t_{2}}\|\nabla u(t)\|_{2}^{2} d t \leq \frac{m_{0}-l}{l \theta} \int_{t_{1}}^{t_{2}} I_{2}(t) d t
\end{aligned}
$$

In the same manner

$$
\begin{aligned}
\int_{0}^{t} \int_{t_{1}}^{t_{2}} g(s)\|\nabla u(t)\|_{2}^{2} d s d t & \leq \int_{0}^{\infty} g(s) d s \int_{t_{1}}^{t_{2}}\|\nabla u(t)\|_{2}^{2} d t \\
& \leq \frac{m_{0}-l}{l \theta} \int_{t_{1}}^{t_{2}} I_{2}(t) d t .
\end{aligned}
$$


Exploiting 3.31 and 3.33) to obtain

$$
\begin{aligned}
& \frac{1}{2} \int_{t_{1}}^{t_{2}}(g o \nabla u)(t) d t=\frac{1}{2} \int_{0}^{t} \int_{t_{1}}^{t_{2}} g(t-s)\|\nabla u(s)-\nabla u(t)\| d s d t \\
& \leq \int_{0}^{t} g(t) d t \int_{t_{1}}^{t_{2}}\|\nabla u(t)\| d t \leq \int_{0}^{t} g(t) d t \int_{t_{1}}^{t_{2}} l\|\nabla u(t)\| d t \\
& \leq \frac{\left(m_{0}-l\right)}{2 \theta} \int_{t_{1}}^{t_{2}} I_{2}(t) d t .
\end{aligned}
$$

It follows by using $(3.8)$ that

$$
\begin{aligned}
& \int_{t_{1}}^{t_{2}} \int_{0}^{1} z^{m+1}(x, k, t) d k d x d t \leq \frac{1}{2 \tau} \int_{t_{1}}^{t_{2}} \int_{t_{1}}^{t_{2}}\left\|u_{t}(s)\right\|_{m+1}^{m+1} d s d \nu \\
& \leq\left(\int_{t_{1}}^{t_{2}} d \nu\right)\left(\int_{t_{1}}^{t_{2}}\left\|u_{t}(s)\right\|_{m+1}^{m+1} d s\right) \leq\left(t_{2}-t_{1}\right) D(t)^{m+1}
\end{aligned}
$$

Using Sobolev's inequality, we get

$$
\begin{aligned}
& \left.\left|\int_{t_{1}}^{t_{2}} \int_{\Omega}\right| u_{t}\right|^{m-1}\left|u_{t}\right| u d x d t \mid \leq \int_{t_{1}}^{t_{2}}\left\|u_{t}\right\|_{m+1}^{m}\|u\|_{m+1} d t \\
& \leq c_{s}\left\|u_{t}\right\|_{m+1}^{m}\|\nabla u\|_{2} d t \leq c_{s}\left(\frac{2(p+1)}{l(p-1)}\right)^{\frac{1}{2}} \sup _{t_{1} \leq s \leq t_{2}}\left((E(s))^{\frac{1}{2}}\right) \\
& \times \int_{t_{1}}^{t_{2}}\left\|u_{t}\right\|_{m+1}^{m} d t \leq c_{s}\left(\frac{2(p+1)}{l(p-1)}\right)^{\frac{1}{2}} E(t)^{\frac{1}{2}}[D(t)]^{m} .
\end{aligned}
$$

Also we have

$$
\begin{aligned}
& \left.\left|\int_{t_{1}}^{t_{2}}\right| z(x, 1, t)\right|^{m-1} z(x, 1, t) u d x d t \mid \leq \int_{t_{1}}^{t_{2}}\|z(t, 1, t)\|_{m+1}^{m}\left\|u_{t}\right\|_{m+1} d t \\
& \leq c_{s}\left(\frac{2(p+1)}{l(p-1)}\right)^{\frac{1}{2}} \sup _{t_{1} \leq s \leq t_{2}}\left((E(s))^{\frac{1}{2}}\right) \int_{t_{1}}^{t_{2}}\|z(x, 1, t)\|_{m+1}^{m} d t \\
& \leq c_{s}\left(\frac{2(p+1)}{l(p-1)}\right)^{\frac{1}{2}}(E(t))^{\frac{1}{2}}[D(t)]^{m} .
\end{aligned}
$$

Therefore, from 3.30-3.37 we deduce

$$
\begin{aligned}
& \int_{t_{1}}^{t_{2}} I_{2}(t) d t \leq 2 c_{s}\left(\frac{2(p+1)}{l(p-1)}\right)^{\frac{1}{2}}(E(t))^{\frac{1}{2}}[D(t)]^{m}+\left(t_{2}-t_{1}\right)[D(t)]^{m+1} \\
& +c(\Omega)[D(t)]^{2}+c_{s} c(\Omega)^{\frac{1}{2}}\left(\frac{2(p+1)}{l(p-1)}\right)^{\frac{1}{2}}(E(t))^{\frac{1}{2}} D(t) \\
& +\left[\frac{\left(2 m_{0}-2 l\right)}{l \theta}+\frac{m_{0}-l}{2 \theta}\right] \int_{t_{1}}^{t_{2}} I_{2}(t) d t
\end{aligned}
$$

Then, rewriting 3.29), we obtain

$$
\begin{aligned}
c_{5} \int_{t_{1}}^{t_{2}} I_{2}(t) d t & \leq c(\Omega)[D(t)]^{2}+c_{3}(E(t))^{\frac{1}{2}} D(t) \\
& +c_{6} E(t)^{\frac{1}{2}}[D(t)]^{m}+c_{4}[D(t)]^{m+1}
\end{aligned}
$$


with

$$
c_{5}=\left[1-\left(\frac{\left(2 m_{0}-2 l\right)}{l \theta}+\frac{m_{0}-l}{2 \theta}\right], c_{3}=c_{s} c(\Omega)\left(\frac{2(p+1)}{l(p-1)}\right)^{\frac{1}{2}}, c_{4}=\left(t_{2}-t_{1}\right), c_{6}=2 c_{5} .\right.
$$

From the condition 3.22 and observing that is equivalent to $c_{5}>0$, then we get

$$
\begin{aligned}
\int_{t_{1}}^{t_{2}} I_{2}(t) d t & \leq c_{7}\left([D(t)]^{2}+(E(t))^{\frac{1}{2}} D(t)+(E(t))^{\frac{1}{2}}[D(t)]^{m}\right. \\
& \left.+[D(t)]^{m+1}\right)
\end{aligned}
$$

where $c_{7}=\frac{\max \left(c_{3}, c_{4}, c_{6}, c(\Omega)\right)}{c_{5}}$. On the other hand, from the definition of $E(t)$ and by 3.15 and () we have

$$
\begin{aligned}
& E(t)=\frac{p-1}{2(p+1)}\left[(g o \nabla u)(t)+\left(m_{0}-\int_{0}^{t} g(s) d s\right)\|\nabla u\|_{2}^{2}\right. \\
& \left.+\int_{\Omega} \int_{0}^{1} z^{m+1}(x, k, s) d k d x\right]+\frac{1}{2}\left\|u_{t}(t)\right\|_{2}^{2}+\frac{\alpha}{2(\gamma+1)}\left(\|\nabla u\|_{2}^{2}\right)^{\gamma+1} \\
& +\frac{1}{p+1} I_{1}(t) \leq \frac{p-1}{2(p+1)}\left[(g o \nabla u)(t)+\left(m_{0}-\int_{0}^{t} g(s) d s\right)\|\nabla u\|_{2}^{2}\right. \\
& \left.+\int_{\Omega} \int_{0}^{1} z^{m+1}(x, k, s) d k d x\right]+\left(\frac{1}{p+1}+\frac{1}{2(\gamma+1)}\right) I_{2}(t) \\
& +\frac{1}{2}\left\|u_{t}(t)\right\|_{2}^{2}
\end{aligned}
$$

where $c_{8}=\frac{1}{p+1}+\frac{1}{2(\gamma+1)}$. Hence, integrating 3.41, over $\left(t_{1}, t_{2}\right)$ and using 3.25, 3.31, 3.34 and 3.39, we deduce that

$$
\begin{aligned}
& \int_{t_{1}}^{t_{2}} E(t) d t \leq \frac{p-1}{2(p+1)} \int_{t_{1}}^{t_{2}}[(g o \nabla u)(t) \\
& \left.+\left(m_{0}-\int_{0}^{t} g(s) d s\right)\|\nabla u\|_{2}^{2}\right] d t \\
& +\frac{p-1}{2(p+1)} \int_{t_{1}}^{t_{2}}\left[\int_{\Omega} \int_{0}^{1} z^{m+1}(x, k, s) d k d x+\right] d t \\
& +\left(\frac{1}{p+1}+\frac{1}{2(\gamma+1)}\right) \int_{t_{1}}^{t_{2}} I_{2}(t) d t+\int_{t_{1}}^{t_{2}} \frac{1}{2}\left\|u_{t}(t)\right\|_{2}^{2} d t \\
& \leq c_{9} \int_{t_{1}}^{t_{2}} I_{2}(t) d t+c_{4}[D(t)]^{m+1}+c(\Omega)[D(t)]^{2} \\
& \leq c_{10}\left[c(\Omega)[D(t)]^{2}+(E(t))^{\frac{1}{2}} D(t)+(E(t))^{\frac{1}{2}}[D(t)]^{m}+[D(t)]^{m+1}\right]
\end{aligned}
$$

where

$$
c_{9}=\frac{p-1}{2(p+1)}\left[\left(\frac{m_{0}-l}{2 \theta}\right)+\frac{1}{\theta}\right]+\frac{1}{p}+\frac{1}{2(\gamma+1)}, c_{10}=\max \left(c_{8}, c(\Omega), c_{4}\right) .
$$

Moreover, integrating 3.42 over $\left(t, t_{2}\right)$, using 3.24 and taking into account the fact that $E\left(t_{2}\right) \leq 2 \int_{t_{1}}^{t_{2}} E(t) d t$, 
due to $t_{2}-t_{1} \geq \frac{1}{2}$, we obtain

$$
\begin{aligned}
& E(t)=E\left(t_{2}\right)+\int_{t}^{t_{2}} \frac{1}{2}\left(g^{\prime} o \nabla u\right)(t) d t+\int_{t}^{t_{2}} \frac{1}{2} g(t)\|\nabla u(t)\|_{2}^{2} d t \\
& +\int_{t}^{t_{2}}\left(\mu_{1}-\frac{\zeta}{\tau}-\frac{\mu_{1}}{m+1}\right)\left\|u^{\prime}(t)\right\|_{m+1}^{m+1} d t \\
& +\int_{t}^{t_{2}}\left(\frac{\zeta}{\tau}-\frac{\mu_{2} m}{m+1}\right) \int_{\Omega} z^{m+1}(x, 1, t) d x d t \\
& \leq 2 \int_{t_{1}}^{t_{2}} E(t) d t+[D(t)]^{m+1}
\end{aligned}
$$

which implies that

$$
E(t) \leq c_{11}\left[[D(t)]^{2}+[E(t)]^{\frac{1}{2}} D(t)+E(t)^{\frac{1}{2}}[D(t)]^{m}+[D(t)]^{m+1}\right] .
$$

Then a simple application of Young's inequality gives, for all $t \geq 0$

$$
E(t) \leq c_{12}\left[[D(t)]^{2}+[D(t)]^{2 m}+[D(t)]^{m+1}\right],
$$

where $c_{11}, c_{12}$ are some positive constants. Therefore, we have the following decay estimate:

(i) If $m=1$. From 3.45, we get

$$
E(t) \leq c_{12}[E(t)-E(t+1)]
$$

here we choose $c_{12}>1$. Thus by Lemma 2.2, we obtain

$$
E(t) \leq E(0) e^{-\tau_{1} t} \quad \text { for } \quad t \geq 0
$$

with $\tau_{1}=\ln \left(\frac{c_{12}}{c_{12}-1}\right)$.

(ii) If $m>1$. It follows from 3.45 that, for all $t \geq 0$,

$$
E(t) \leq c_{12}\left(\left(1+[D(t)]^{m-1}+[D(t)]^{2 m-2}\right)[D(t)]^{2}\right) .
$$

As

$$
D(t) \leq E(t)^{\frac{1}{m+1}} \leq E(0)^{\frac{1}{m+1}}
$$

then by (3.45) and (3.46), we have, for all $t \geq 0$

$$
E(t) \leq c_{11}\left[\left(1+E(0)^{\frac{m-1}{m+1}}+E(0)^{\frac{2 m-2}{m+1}}\right)\right][D(t)]^{2} \leq c_{12}(E(0))[D(t)]^{2},
$$

where $c_{12}=\left(\frac{1}{E(0)}+E(0)^{\frac{-2}{m+1}}+E(0)^{\frac{m-3}{m+1}}\right)$. We get easily that

$$
\begin{aligned}
(E(t))^{1+m} & \leq\left[c_{13}(E(0)) D(t)^{2}\right]^{1+m} \leq c_{14}(E(0))[D(t)]^{2 m+2} \\
& \leq c_{14} E(0)\left[(D(t)]^{m+1}=c_{15}(E(0))(E(t)-E(t+1)),\right.
\end{aligned}
$$

where

$$
c_{14}(E(0))=2^{m}\left(c_{13}(E(0))\right)^{m+1}, c_{15}(E(0))=c_{14}(E(0)) E(0) .
$$

Applying the Lemma 2.2 to 3.49 yields

$$
\phi(t) \leq\left(\phi(0)^{-m}+\tau_{2} m[t-1]^{+}\right)^{\frac{-1}{m}}, \quad \text { for } \quad t \geq 0,
$$

with $\tau_{2}=c_{14}^{-1}(E(0))$. The proof of Theorem 3.1 is completed. 


\section{Blow up result}

In this section we have a condition on parameters $m$ and $p$ as follows

$$
\frac{n}{(n-2)}>p>m \geq 1, n \geq 3(1<p, n \leq 2) .
$$

2- Nonlinear case: $m>1$, with $E(0)<0$.

For this case our result reads as follows:

Theorem 4.1. Let $u_{0} \in H_{0}^{1}(\Omega) \cap H^{2}(\Omega), u_{1} \in H_{0}^{1}(\Omega)$ and $f_{0} \in L^{2}(\Omega \times(0,1))$ be given. Assume that the assumptions $\left(A_{0}\right)-\left(A_{2}\right)$ are fulfilled. Let $p>2$ and suppose that (4.1) holds. Then for any initial data satisfying $E(0)<0$, the solution of (3.1) blows up in finite time.

Proof. We set

$$
H(t)=-E(t) .
$$

Using the Lemma 3.1, to get

$$
\begin{aligned}
& H^{\prime}(t) \geq\left(\mu_{1}-\frac{\zeta}{\tau}-\frac{\mu_{2}}{m+1}\right)\left\|u_{t}\right\|_{m}^{m}+\frac{1}{2} g(t)\|\nabla u\|_{2}^{2} \\
& +\left(\frac{\zeta}{\tau}-\frac{\mu_{2} m}{m+1}\right) \int_{\Omega} z^{m+1}(x, 1, t) d x-\frac{1}{2}\left(g^{\prime} o \nabla u\right)(s) d s
\end{aligned}
$$

hence,

$$
0<H(0) \leq H(t) \leq \frac{1}{p+1}\|u\|_{p+1}^{p+1}, t>0
$$

Let

$$
M(t)=\|u\|_{2}^{2}
$$

Differentiating (4.5) twice, we obtain

$$
M^{\prime}(t)=2 \int_{\Omega} u_{t} u d x
$$

and

$$
M^{\prime \prime}(t)=2\left\|u_{t}\right\|_{2}^{2}+2 \int_{\Omega} u_{t t} u d x .
$$

Using the first equation in (3.1), to get

$$
\begin{aligned}
& M^{\prime \prime}(t)=2\left\|u_{t}\right\|_{2}^{2}+2 \int_{\Omega} \int_{0}^{t} g(t-s) \nabla u(s) \nabla u(t) d x d s \\
& -2 \mu_{1} \int_{\Omega}\left|u_{t}(x, t)\right|^{m-1} u u_{t}(x, t) d x-2 M\left(\|\nabla u\|_{2}^{2}\right)\|\nabla u\|_{2}^{2} \\
& -2 \mu_{2} \int_{\Omega}|z(x, 1, t)|^{m-1} u z(x, 1, t) d x+2\|u\|_{p+1}^{p+1} .
\end{aligned}
$$

Using Holderś and Young's inequalities to get

$$
\begin{aligned}
& \int_{\Omega} \int_{0}^{t} g(t-s) \nabla u(s) \nabla u(t) d x d s=\int_{0}^{t} g(t-s)\|\nabla u(t)\|_{2}^{2} d s \\
& +\int_{\Omega} \int_{0}^{t} g(t-s) \nabla u(t)(\nabla u(s)-\nabla u(t)) d s d x \\
& \geq-(g o \nabla u)(t)+\frac{3}{4} \int_{0}^{t} g(s) d s\|\nabla u(t)\|_{2}^{2} .
\end{aligned}
$$


Noticing the estimate (4.8), then 4.7) takes the form

$$
\begin{aligned}
& M^{\prime \prime}(t) \geq(p+1)\left\|u_{t}\right\|_{2}^{2}+2(p+1) H(t)+(p-1)(g o \nabla u)(t) \\
& +\frac{(1-2 p)}{2} \int_{0}^{t} g(s) d s\|\nabla u(t)\|_{2}^{2}-2 m_{0}\|\nabla u(t)\|_{2}^{2}-2 \alpha\left(\|\nabla u(t)\|_{2}^{2}\right)^{\gamma+1} \\
& +(p+1) m_{0}\|\nabla u(t)\|_{2}^{2}+\frac{(p+1) \alpha}{\gamma+1}\left(\|\nabla u(t)\|_{2}^{2}\right)^{\gamma+1} \\
& +m_{1}\|\nabla u\|_{2}^{2}-2 \mu_{1} \int_{\Omega}\left|u_{t}(x, t)\right|^{m-1} u u^{\prime}(x, t) d x \\
& -2 \mu_{2} \int_{\Omega}|z(x, 1, t)|^{m-1} u z(x, 1, t) d x \\
& +(p+1) \zeta \int_{\Omega} \int_{0}^{1} z^{m+1}(x, k, t) d k d x .
\end{aligned}
$$

Using (2.1) to get

$$
\begin{aligned}
& M^{\prime \prime}(t) \geq(p+1)\left\|u_{t}\right\|_{2}^{2}+2(p+1) H(t)+(p-1)(g o \nabla u)(t) \\
& -2 \mu_{1} \int_{\Omega}\left|u_{t}(x, t)\right|^{m-1} u u^{\prime}(x, t) d x \\
& -2 \mu_{2} \int_{\Omega}|z(x, 1, t)|^{m-1} u z(x, 1, t) d x \\
& +(p+1) \zeta \int_{\Omega} \int_{0}^{1} z^{m+1}(x, k, t) d k d x+m_{1}\|\nabla u\|_{2}^{2}
\end{aligned}
$$

Now we define the functional

$$
L(t)=H(t)^{1-\kappa}+2 \epsilon M^{\prime}(t)
$$

Differentiating (4.11), we obtain

$$
L^{\prime}(t)=(1-\kappa) H(t)^{-\kappa} H^{\prime}(t)+2 \varepsilon M^{\prime \prime}(t) .
$$

Replacing 4.10 in 4.12, we get

$$
\begin{aligned}
& L^{\prime}(t) \geq(1-\kappa) H(t)^{-\kappa} H^{\prime}(t)+2 \varepsilon(p+1)\left\|u^{\prime}\right\|_{2}^{2}+2 \varepsilon(p+1) H(t) \\
& -2 \varepsilon \mu_{1} \int_{\Omega}\left|u^{\prime}(x, t)\right|^{m-1} u u^{\prime}(x, t) d x+m_{1}\|\nabla u\|_{2}^{2} \\
& -2 \varepsilon \mu_{2} \int_{\Omega}|z(x, 1, t)|^{m-1} u z(x, 1, t)+\varepsilon(p-1)(g o \nabla u)(t) d x \\
& +\varepsilon(p+1) \zeta \int_{\Omega} \int_{0}^{1} z^{m+1}(x, k, t) d k d x .
\end{aligned}
$$

Note that by 4.4 and using Holder inequality, we obtain

$$
\begin{aligned}
\left.\left|\int_{\Omega} u\right| u_{t}\right|^{m-1} u_{t} d x \mid & \leq\left\|u_{t}\right\|_{m+1}^{m}\|u\|_{m} \leq c_{1}\|u\|_{m+1}^{\frac{m+1}{p+1}}\|u\|_{m+1}^{1-\frac{m+1}{p+1}}\left\|u_{t}\right\|_{m+1}^{m} \\
& \leq c_{2}\|u\|_{m+1}^{\frac{m+1}{p+1}} H(t)^{\frac{1}{p+1}-\frac{m+1}{(p+1)^{2}}}\left\|u_{t}\right\|_{m+1}^{m},
\end{aligned}
$$

By Young's inequality and 4.3), produces

$$
\begin{aligned}
\left.\left|\int_{\Omega} u\right| u_{t}\right|^{m-1} u_{t} d x \mid & \leq c_{3}\left(\rho^{\frac{1}{1+p}}\|u\|_{m+1}^{m+1} H(0)^{-\bar{k}}\right. \\
& \left.+\rho^{-m^{\prime}} H(0)^{k-\bar{k}} H^{\prime}(t) H(t)^{-k}\right)
\end{aligned}
$$


where $\bar{k}=\frac{1}{p+1}-\frac{m+1}{(p+1)^{2}}>0, \rho>0, m^{\prime}=\frac{m+1}{m}$. Letting $0<k<\bar{k}$. Similarly we have

$$
\begin{aligned}
\left.\left|\int_{\Omega} u\right| z(x, 1, t)\right|^{m-1} z(x, 1, t) d x \mid & \leq c_{3}\left(\rho^{\frac{1}{1+p}}\|u\|_{m+1}^{m+1} H(0)^{-\bar{k}}\right. \\
& \left.+\rho^{-m^{\prime}} H(0)^{k-\bar{k}} H^{\prime}(t) H(t)^{-k}\right) .
\end{aligned}
$$

Exploiting 4.14 and 4.15, to get

$$
\begin{aligned}
& L^{\prime}(t) \geq\left[(1-k)-2 \epsilon\left(\mu_{1}+\mu_{2}\right) H^{k-\bar{k}}(0) \rho^{-m^{\prime}}\right] H(t)^{-k} H^{\prime}(t) \\
& -2 \epsilon\left(\mu_{1}+\mu_{2}\right) H(0)^{-\bar{k}} \rho^{\frac{1}{p+1}}\|u\|_{m+1}^{m+1}+\epsilon m_{1}\|\nabla u\|_{2}^{2}+2 \epsilon(p+1) H(t) \\
& +\epsilon(p+2)\left\|u_{t}\right\|_{2}^{2}+\epsilon(p-1)(g o \nabla u)(t) \\
& +\epsilon(p+1) \zeta \int_{\Omega} \int_{0}^{1} z^{m+1}(x, k, t) d k d x,
\end{aligned}
$$

for $\epsilon$ sufficiently small, we have

$$
\left[(1-k)-2 \epsilon\left(\mu_{1}+\mu_{2}\right) H^{k-\bar{k}}(0) \rho^{-m^{\prime}}\right] \geq 0 .
$$

We set $s=m+1 \leq p+1$ such that $\|u\|_{m+1}^{s} \leq c_{1}\left(\|\nabla u\|_{2}^{2}+\|u\|_{p+1}^{p+1}\right)$, where $c=2\left(\mu_{1}+\mu_{2}\right) H(0)^{-\bar{k}} \rho^{\frac{1}{p+1}} c_{1}$ and taking $m_{1}>c$. Then

$$
\begin{aligned}
L^{\prime}(t) & \geq \epsilon\left(m_{1}-c\right)\|\nabla u\|_{2}^{2}-\epsilon c\|u\|_{p+1}^{p+1}+2 \epsilon(p+1) H(t)+\epsilon(p+2)\left\|u_{t}\right\|_{2}^{2} \\
& +\epsilon(p-1)(g o \nabla u)(t)+\epsilon(p+1) \zeta \int_{\Omega} \int_{0}^{1} z^{m+1}(x, k, t) d k d x .
\end{aligned}
$$

Using this notation $a_{1}=m_{1}-c, a_{2}=c, a_{3}=2(p+1), a_{4}=p+2, a_{5}=p-1$, then 4.18 becomes

$$
\begin{aligned}
L^{\prime}(t) & \geq a_{1} \epsilon\|\nabla u\|_{2}^{2}-\epsilon a_{2}\|u\|_{p+1}^{p+1}+\epsilon a_{3} H(t)+\epsilon a_{4}\left\|u_{t}\right\|_{2}^{2} \\
& +\epsilon a_{5}(g o \nabla u)(t) .
\end{aligned}
$$

Following the approach of Messaoudi [13] we can suppose that $p=2 a_{6}+\left(p-2 a_{6}\right)$, where $a_{6}<$ $\min \left(a_{1}, a_{2}, a_{3}, a_{4}, a_{5}\right)$, then 4.19 takes the form

$$
\begin{aligned}
L^{\prime}(t) & \geq\left(a_{1}-a_{6}\right) \epsilon\|\nabla u\|_{2}^{2}+\epsilon\left(a_{6}-a_{2}\right)\|u\|_{p+1}^{p+1}+\epsilon\left(a_{3}-a_{6}\right) H(t) \\
& +\epsilon\left(a_{4}-a_{6}\right)\left\|u_{t}\right\|_{2}^{2}+\epsilon\left(a_{5}-a_{6}\right)(g o \nabla u)(t),
\end{aligned}
$$

it produces

$$
L^{\prime}(t) \geq \delta \epsilon\left[\|\nabla u\|_{2}^{2}+\|u\|_{p+1}^{p+1}+H(t)+\left\|u_{t}\right\|_{2}^{2}+(g o \nabla u)(t)\right]
$$

finally

$$
L^{\prime}(t) \geq \delta \epsilon\left[\|u\|_{p+1}^{p+1}+H(t)+\left\|u_{t}\right\|_{2}^{2}\right],
$$

where $\delta>0$ is the minimum of the coefficients of $\|u\|_{p+1}^{p+1}, H(t),\left\|u_{t}\right\|_{2}^{2}$. We pick out $\epsilon$ so that

$$
L(0)=H(t)^{1-\kappa}(0)+2 \epsilon \int_{\Omega} u^{1} u^{0} d x>0 .
$$

Consequently we have

$$
L(t) \geq L(0)>0, \quad \forall t \geq 0 .
$$


Now, we set $\varpi=\frac{1}{1-k}$, and since $k<\bar{k}<1$ it is not difficult to see that $1<\varpi<\frac{1}{1-\bar{k}}$. We set

$$
L(t)=H(t)^{1-k}+2 \epsilon \int_{\Omega} u_{t} u d x
$$

then

$$
L(t)=H(t)^{\frac{1}{\varpi}}+2 \epsilon \int_{\Omega} u_{t} u d x \leq H(t)^{\frac{1}{\varpi}}+2 \epsilon \int_{\Omega} u_{t} u d x .
$$

By using Young, Holder's inequalities and 4.26 ) it follows that

$$
\begin{aligned}
& L(t)^{\varpi} \leq H(t)+\left[2 \epsilon \int_{\Omega} u_{t} u d x+2 E_{1}\left(\|u\|_{p+1}\right)^{\frac{p+1}{\varpi}}\right]^{\varpi} \\
& \leq 2^{\varpi-1}\left[H(t)+2^{\varpi-1}\left(\beta^{\varpi}\left\|u_{t}\right\|_{2}^{\varpi}\|u\|_{2}^{\varpi}\right) \leq c_{2}\left[H(t)+\left\|u_{t}\right\|_{2}^{\varpi}\|u\|_{2}^{\varpi}\right],\right.
\end{aligned}
$$

where $c_{2}=\max \left\{2^{\varpi-1}, \beta^{\varpi}\right\}$. On the other hand for $p>1$, using Holder and Young inequalities we have

$$
\left\|u_{t}\right\|_{2}^{\varpi}\|u\|_{2}^{\varpi} \leq c_{3}\left\|u_{t}\right\|_{2}^{\varpi}\|u\|_{p+1}^{\varpi} \leq c_{4}\left(\left\|u_{t}\right\|_{2}^{2}+\|u\|_{p+1}^{\frac{2}{1-2 k}}\right)
$$

and

$$
\|u\|_{p+1}^{\frac{2}{1-2 k}}=\|u\|_{p+1}^{p+1}\|u\|_{2}^{\frac{2}{1-2 k}-\frac{1}{p+1}} \leq c_{5} H(0)^{\frac{2}{(1-2 k)(p+1)}-1}\|u\|_{p+1}^{p+1} .
$$

Then 4.27) takes the form

$$
L(t)^{\varpi} \leq c_{6}\left(H(t)+\left\|u_{t}\right\|_{2}^{2}+\|u\|_{p+1}^{p+1}\right) .
$$

Combining (4.22) and 4.30) we deduce that

$$
L^{\prime}(t) \geq c_{7} L(t)^{\varpi}, c_{7}>0, \varpi>1 .
$$

A simple integration over $(0, t)$ yields the desired result.

3- Nonlinear case: $m>1$, with $E(0)>0$.

In this subsection, we investigate the blow up of solutions with nonlinear terms and for positive initial energy $E(0)>0$. For this purpose, we define the functional

$$
G(x)=\frac{1}{2} x^{2}-\frac{B}{p+1} x^{p+1} .
$$

Moreover, we introduce the following notations

$$
B=c_{s}^{p+1} l, \lambda_{1}=c_{s}^{-\frac{p+1}{p-2}}, E_{1}=\left(\frac{1}{2}-\frac{1}{p+1}\right) \lambda_{1},
$$

where

$$
\begin{aligned}
\varphi(t)=\left[l\|\nabla u\|_{2}^{2}+(g o \nabla u)(t)\right. & +\frac{\alpha}{\gamma+1}\left(\|\nabla u\|_{2}^{2}\right)^{\gamma+1} \\
& \left.+\zeta \int_{0}^{1} \int_{\Omega} z^{m+1}(x, k, t) d k d x\right]^{\frac{1}{2}} .
\end{aligned}
$$


Lemma 4.2. Assume $\left(A_{1}\right)$ holds. Let $(u, z)$ be a solution of (3.1) with initial data satisfying $E(0)<E_{1}$ and $\varphi(0)>\lambda_{1} i, e$.

$$
\begin{aligned}
{\left[l\|\nabla u(0)\|_{2}^{2}\right.} & +\frac{\alpha}{\gamma+1}\left(\|\nabla u(0)\|_{2}^{2}\right)^{\gamma+1} \\
& \left.+\zeta \int_{0}^{1} \int_{\Omega} z^{m+1}(x, k, 0) d k d x\right]^{\frac{1}{2}}>\lambda_{1} .
\end{aligned}
$$

Then there exists $\lambda_{2}>\lambda_{1}$ such that, for all $t \geq 0$

$$
\varphi(t) \geq \lambda_{2}, \quad \text { and } \quad\|u\|_{p+1}^{p+1} \geq \lambda_{3}=c_{s}^{p+1} \lambda_{2}^{p+1},
$$

Proof. As in [6] but here we give slight modification. We observe that $G$ is increasing for $0<\lambda<\lambda_{1}$, decreasing for $\lambda>\lambda_{1}$ and $G(\lambda) \rightarrow-\infty$ as $\lambda \rightarrow \infty$. Thus, as $E(0)<E_{1}$, there exist $\lambda_{2}^{\prime}<\lambda_{1}<\lambda_{2}$ such that $G\left(\lambda_{2}^{\prime}\right)=G\left(\lambda_{2}\right)=E(0)$, which together with $\varphi(0) \geq \lambda_{1}$ and 4.32 infer that

$$
G(\varphi(0)) \leq E(0)=G\left(\lambda_{2}\right) .
$$

This implies that $\varphi(0) \geq \lambda_{2}$. To establish the first estimate in (4.36), we argue by contradiction. Suppose that the first estimate in (4.36) does not hold, then there exists $t^{*}>0$ such that

$$
\varphi\left(t^{*}\right)<\lambda_{2} .
$$

We easily observe from (3.14) and (3.15) and Lemma 3.2 that

$$
\begin{aligned}
& E(t) \geq J(t)=\frac{1}{2} \varphi(t)^{2}-\frac{1}{p+1}\|u\|_{p+1}^{p+1} \geq \frac{1}{2} \varphi(t)^{2} \\
& -\frac{1}{p+1} c_{s}^{p+1} l\|\nabla u\|_{2}^{p+1} \geq \frac{1}{2} \varphi(t)^{2}-\frac{c_{s}^{p+1} l}{p+1} \varphi(t)^{p+1}=G(\varphi(t)) .
\end{aligned}
$$

Case 1: if $\lambda_{1}<\varphi\left(t^{*}\right)<\lambda_{2}$, then

$$
G\left(\varphi\left(t^{*}\right)\right)>G\left(\lambda_{2}\right)=E(0) \geq E\left(t^{*}\right) .
$$

This contradicts the first estimate in 4.36.

Case 2: if $\varphi\left(t^{*}\right) \leq \lambda_{1}$, then by continuity of the function $\varphi(t)$, there exists $0<t_{1}<t^{*}$ such that

$$
\lambda_{1}<\varphi\left(t_{1}\right)<\lambda_{2},
$$

then

$$
G\left(\varphi\left(t_{1}\right)\right)>G\left(\lambda_{2}\right)=E(0) \geq E\left(t_{1}\right) .
$$

This is also a contradiction of the first estimate of (4.36). Thus, we have proved the first estimate in 4.36 ). To prove the second estimate in 4.36 we have

$$
\begin{aligned}
& E(t)=\frac{1}{2}\left\|u^{\prime}(t)\right\|_{2}^{2}+\frac{1}{2}(g o \nabla u)(t)+\frac{1}{2}\left(m_{0}-\int_{0}^{t} g(s) d s\right)\|\nabla u\|_{2}^{2} \\
& -\frac{1}{p+1}\|u\|_{p+1}^{p+1}+\frac{\alpha}{2(\gamma+1)}\left(\|\nabla u\|_{2}^{2}\right)^{\gamma+1}+\frac{\zeta}{2} \int_{\Omega} \int_{0}^{1} z^{m+1}(x, k, s) d k d x \\
& \leq E(0) .
\end{aligned}
$$

Hence

$$
-\frac{1}{p+1}\|u\|_{p+1}^{p+1}+\frac{1}{2}\left\|u_{t}\right\|_{2}^{2}+\varphi(t)^{2} \leq E(0)
$$


Then from 4.38 we have

$$
\begin{aligned}
\frac{1}{p+1}\|u\|_{p+1}^{p+1} \geq \varphi(t)^{2}-E(0) \geq \frac{1}{2} \lambda_{2}^{2}-E(0) & =\frac{1}{2} \lambda_{2}^{2}-G\left(\lambda_{2}\right) \\
& =\frac{c_{s}^{p+1}}{p+1} \lambda_{2}^{p+1} .
\end{aligned}
$$

This completes the proof.

Theorem 4.3. Let $u_{0} \in H_{0}^{1}(\Omega) \cap H^{2}(\Omega), u_{1} \in H_{0}^{1}(\Omega)$ and $f_{0} \in L^{2}(\Omega \times(0,1))$ be given. Assume that the hypotheses $\left(A_{0}\right)-\left(A_{2}\right)$ hold, for any initial data satisfying $E(0)<E_{1}, \varphi(0)>\lambda_{1}$. Then any solution of problem (3.1) blows up at finite time $T$.

Proof. We set

$$
H(t)=E_{1}-E(t), t \geq 0 .
$$

By Lemma 3.1, we have

$$
\begin{aligned}
& H^{\prime}(t)=-E^{\prime}(t)=\left(\mu_{1}-\frac{\zeta}{2 \tau}-\frac{\mu_{1}}{m+1}\right)\left\|u_{t}\right\|_{m+1}^{m+1} \\
& +\left(\frac{\zeta}{\tau}-\frac{\mu_{2} m}{m+1}\right) \int_{\Omega} z^{m+1}(x, 1, t) d x-\frac{1}{2}\left(g^{\prime} o \nabla u\right)(t)+\frac{1}{2} g(t)\|\nabla u(t)\|_{2}^{2},
\end{aligned}
$$

consequently

$$
\begin{aligned}
& 0<E_{1}-E(0)=H(0) \leq H(t)=E_{1}-\frac{1}{2} \varphi(t)^{2}+\frac{1}{p+1}\|u\|_{p+1}^{p+1} \\
& \leq-\frac{1}{p+1} \lambda_{1}^{2}+\frac{1}{p}\|u\|_{p+1}^{p+1} \leq \frac{1}{p+1}\|u\|_{p+1}^{p+1}, \quad \forall t \geq 0 .
\end{aligned}
$$

Now we define the functional

$$
L(t)=H(t)^{1-\kappa}+2 \epsilon \int_{\Omega} u_{t} u d x+2 t E_{1}
$$

Differentiating (4.44), to obtain

$$
L^{\prime}(t)=(1-\kappa) H(t)^{-\kappa} H^{\prime}(t)+2 \epsilon \int_{\Omega} u_{t t} u d x+2 \epsilon\left\|u_{t}\right\|_{2}^{2}+2 E_{1} .
$$

Then from 4.45 we have

$$
\begin{aligned}
& L^{\prime}(t)=(1-\kappa) H(t)^{-\kappa} H^{\prime}(t)+2 \epsilon\left\|u_{t}\right\|_{2}^{2}-2 \epsilon\|\nabla u\|_{2}^{2} \\
& -2 \epsilon \alpha\left(\|\nabla u\|_{2}^{2}\right)^{\gamma+1}+2 \epsilon \int_{\Omega} \int_{0}^{t} g(t-s) \nabla u(s) \nabla u(t) d x d s \\
& -2 \epsilon \mu_{1} \int_{\Omega}\left|u_{t}(x, t)\right|^{m-1} u u_{t}(x, t) d x \\
& -2 \epsilon \mu_{2} \int_{\Omega}|z(x, 1, t)|^{m-1} u z(x, 1, t) d x+2 \epsilon\|u\|_{p+1}^{p+1}+2 E_{1} .
\end{aligned}
$$

Using Holder and Young's inequalities, to get

$$
\begin{aligned}
& \int_{\Omega} \int_{0}^{t} g(t-s) \nabla u(s) \nabla u(t) d x d s=\int_{\Omega} \int_{0}^{t} g(t-s) \nabla u(t)(\nabla u(s) \\
& -\nabla u(t)) d s d x+\int_{0}^{t} g(t-s) d s\|\nabla u(t)\|_{2}^{2} \\
& \geq-(g o \nabla u)(t)+\frac{3}{4} \int_{0}^{t} g(s) d s\|\nabla u(t)\|_{2}^{2} .
\end{aligned}
$$


Using $\left(A_{2}\right)$ then 4.46 takes the form

$$
\begin{aligned}
& L^{\prime}(t)=(1-\kappa) H(t)^{-\kappa} H^{\prime}(t)+2 \epsilon(1+p)\left\|u_{t}\right\|_{2}^{2}+\epsilon a_{1}(g o \nabla u)(t) \\
& +2 E_{1} a_{2}+2 \epsilon(p+1) H(t)-2 \epsilon \mu_{1} \int_{\Omega}\left|u_{t}(x, t)\right|^{m-1} u u_{t}(x, t) d x \\
& -2 \epsilon \mu_{2} \int_{\Omega}|z(x, 1, t)|^{m-1} u z(x, 1, t) d x \\
& +(p+1) \epsilon \zeta \int_{\Omega} \int_{0}^{1} z^{m+1}(x, k, t) d k d x+m_{1} \epsilon\|\nabla u\|_{2}^{2},
\end{aligned}
$$

for $\epsilon$ sufficiently small and, $p>1, a_{1}=(p-1), a_{2}=(1-\epsilon p)>0$, this yields

$$
\begin{aligned}
& L^{\prime}(t) \geq(1-\kappa) H(t)^{-\kappa} H^{\prime}(t)+2 \epsilon(1+p)\left\|u_{t}\right\|_{2}^{2}+\epsilon a_{1}(g o \nabla u)(t) \\
& +\epsilon m_{1}\|\nabla u\|_{2}^{2}-2 \epsilon \mu_{1} \int_{\Omega}\left|u_{t}(x, t)\right|^{m-1} u u_{t}(x, t) d x \\
& -2 \epsilon \mu_{2} \int_{\Omega}|z(x, 1, t)|^{m-1} u z(x, 1, t) d x+2 \epsilon(p+1) H(t) \\
& +(p+1) \epsilon \zeta \int_{\Omega} \int_{0}^{1} z^{m+1}(x, k, t) d k d x .
\end{aligned}
$$

Using Holder inequality and 4.42, we obtain

$$
\begin{aligned}
& \left.\left|\int_{\Omega} u\right| u_{t}\right|^{m-1} u_{t} d x \mid \leq\left\|u_{t}\right\|_{m+1}^{m}\|u\|_{m} \leq c_{1}\|u\|_{m+1}^{\frac{m+1}{p+1}}\|u\|_{m+1}^{1-\frac{m+1}{p+1}}\left\|u_{t}\right\|_{m+1}^{m} \\
& \leq c_{2}\|u\|_{m+1}^{\frac{m+1}{p+1}} H(t)^{\frac{1}{p+1}-\frac{m+1}{(p+1)^{2}}}\left\|u_{t}\right\|_{m+1}^{m} .
\end{aligned}
$$

By Young's inequality and 4.42, we obtain

$$
\begin{aligned}
\left.\left|\int_{\Omega} u\right| u_{t}\right|^{m-1} u_{t} d x \mid & \leq c_{3}\left(\gamma^{\frac{1}{1+p}}\|u\|_{m+1}^{m+1} H(0)^{-\bar{k}}\right. \\
& \left.+\gamma^{-m^{\prime}} H(0)^{k-\bar{k}} H^{\prime}(t) H(t)^{-k}\right),
\end{aligned}
$$

where $\bar{k}=\frac{1}{p+1}-\frac{m+1}{(p+1)^{2}}>0, \gamma>0, m^{\prime}=\frac{m+1}{m}$. Letting $0<k<\bar{k}$. Similarly we have

$$
\begin{aligned}
\left.\left|\int_{\Omega} u\right| z(x, 1, t)\right|^{m-1} z(x, 1, t) d x \mid & \leq c_{3}\left(\gamma^{\frac{1}{1+p}}\|u\|_{m+1}^{m+1} H(0)^{-\bar{k}}\right. \\
& \left.+\gamma^{-m^{\prime}} H(0)^{k-\bar{k}} H^{\prime}(t) H(t)^{-k}\right) .
\end{aligned}
$$

Exploiting 4.51 and 4.52, to get

$$
\begin{aligned}
& L^{\prime}(t) \geq\left[(1-k)-2 \epsilon\left(\mu_{1}+\mu_{2}\right) H^{k-\bar{k}}(0) \gamma^{-m^{\prime}}\right] H(t)^{-k} H^{\prime}(t) \\
& -2 \epsilon\left(\mu_{1}+\mu_{2}\right) H(0)^{-\bar{k}} \gamma^{\frac{1}{p+1}}\|u\|_{m+1}^{m+1}+\epsilon m_{1}\|\nabla u\|_{2}^{2}+2 \epsilon(p+1) H(t) \\
& +2 \epsilon(p+1)\left\|u_{t}\right\|_{2}^{2}+\epsilon(p-1)(g o \nabla u)(t) \\
& +\epsilon(p+1) \zeta \int_{\Omega} \int_{0}^{1} z^{m+1}(x, k, t) d k d x,
\end{aligned}
$$

for $\epsilon$ sufficiently small such that

$$
\left[(1-k)-2 \epsilon\left(\mu_{1}+\mu_{2}\right) H^{k-\bar{k}}(0) \gamma^{-m^{\prime}}\right] \geq 0
$$


We set $s=m+1 \leq p+1$ such that $\|u\|_{m+1}^{s} \leq c_{1}\left(\|\nabla u\|_{2}^{2}+\|u\|_{p+1}^{p+1}\right)$, where $c=2\left(\mu_{1}+\mu_{2}\right) H(0)^{-\bar{k}} \gamma^{\frac{1}{p+1}} c_{1}$ and taking $m_{1}>c$, we arrive at

$$
\begin{aligned}
& L^{\prime}(t) \geq \epsilon\left(m_{1}-c\right)\|\nabla u\|_{2}^{2}-\epsilon c\|u\|_{p+1}^{p+1}+2 \epsilon(p+1) H(t) \\
& +2 \epsilon(p+1)\left\|u_{t}\right\|_{2}^{2}+\epsilon(p-1)(g o \nabla u)(t) \\
& +\epsilon(p+1) \zeta \int_{\Omega} \int_{0}^{1} z^{m+1}(x, k, t) d k d x .
\end{aligned}
$$

Using this notation $a_{1}=m_{1}-c, a_{2}=c, a_{3}=2(p+1), a_{4}=p+2, a_{5}=p-1$, then 4.54 becomes

$$
\begin{aligned}
L^{\prime}(t) & \geq a_{1} \epsilon\|\nabla u\|_{2}^{2}-\epsilon a_{2}\|u\|_{p+1}^{p+1}+\epsilon a_{3} H(t)+\epsilon a_{4}\left\|u_{t}\right\|_{2}^{2} \\
& +\epsilon a_{5}(g o \nabla u)(t) .
\end{aligned}
$$

Following the approach of Messaoudi [13] we can suppose that $p=2 a_{6}+\left(p-2 a_{6}\right)$, where $a_{6}<$ $\min \left(a_{1}, a_{2}, a_{3}, a_{4}, a_{5}\right)$ then 4.55 takes the form

$$
\begin{aligned}
L^{\prime}(t) & \geq\left(a_{1}-a_{6}\right) \epsilon\|\nabla u\|_{2}^{2}+\epsilon\left(a_{6}-a_{2}\right)\|u\|_{p+1}^{p+1}+\epsilon\left(a_{3}-a_{6}\right) H(t) \\
& +\epsilon\left(a_{4}-a_{6}\right)\left\|u_{t}\right\|_{2}^{2}+\epsilon\left(a_{5}-a_{6}\right)(g o \nabla u)(t)
\end{aligned}
$$

hence

$$
L^{\prime}(t) \geq \delta \epsilon\left[\|\nabla u\|_{2}^{2}+\|u\|_{p+1}^{p+1}+H(t)+\left\|u_{t}\right\|_{2}^{2}+(g o \nabla u)(t)\right]
$$

finally

$$
L^{\prime}(t) \geq \delta \epsilon\left[\|u\|_{p+1}^{p+1}+H(t)+\left\|u_{t}\right\|_{2}^{2}\right]
$$

where $\delta>0$ is the minimum of the coefficients of $\|u\|_{p+1}^{p+1}, H(t),\left\|u_{t}\right\|_{2}^{2}$. We pick out $\epsilon$ so that

$$
L(0)=H(t)^{1-\kappa}(0)+2 \epsilon \int_{\Omega} u^{1} u^{0} d x>0 .
$$

Consequently we have

$$
L(t) \geq L(0)>0, \quad \forall t \geq 0 .
$$

Now, we set $\varpi=\frac{1}{1-k}$, and since $k<\bar{k}<1$ it is not hard to see that $1<\varpi<\frac{1}{1-\bar{k}}$. We set

$$
L(t)=H(t)^{1-k}+2 \epsilon \int_{\Omega} u_{t} u d x+2 t \epsilon E_{1} .
$$

Taking into account 4.36 of Lemma 4.1 we also can choose $\epsilon$ small enough such that $\epsilon t<\left(\lambda_{3}\right)^{\frac{p+1}{\varpi}}$, we obtain

$$
\begin{aligned}
L(t) & =H(t)^{\frac{1}{\varpi}}+2 \epsilon \int_{\Omega} u_{t} u d x+2 E_{1}\left(\lambda_{3}\right)^{\frac{p+1}{\varpi}} \\
& \leq H(t)^{\frac{1}{\varpi}}+2 \epsilon \int_{\Omega} u_{t} u d x+2 E_{1}\left(\|u\|_{p+1}\right)^{\frac{p+1}{\varpi}} .
\end{aligned}
$$

By using Young, Holder's inequalities and 4.61) it follows that

$$
\begin{aligned}
L(t)^{\varpi} & \leq H(t)+\left[2 \epsilon \int_{\Omega} u_{t} u d x+2 E_{1}\left(\|u\|_{p+1}\right)^{\frac{p+1}{\varpi}}\right]^{\varpi} \\
& \leq 2^{\varpi-1}\left[H(t)+2^{\varpi-1}\left(\beta^{\varpi}\left\|u_{t}\right\|_{2}^{\varpi}\|u\|_{2}^{\varpi}+\left(2 E_{1}\right)^{\varpi}\|u\|_{p+1}^{p+1}\right)\right. \\
& \leq c_{2}\left(H(t)+\left\|u_{t}\right\|_{2}^{\varpi}\|u\|_{2}^{\varpi}+\|u\|_{p+1}^{p+1}\right)
\end{aligned}
$$


where $c_{2}=\max \left\{2^{\varpi-1}, \beta^{\varpi},\left(2 E_{1}\right)^{\varpi}\right\}$. On the other hand, for $p>1$, using Holder and Young inequalities we have

$$
\begin{gathered}
\left\|u_{t}\right\|_{2}^{\varpi}\|u\|_{2}^{\varpi} \leq c_{3}\left\|u_{t}\right\|_{2}^{\varpi}\|u\|_{p+1}^{\varpi} \leq c_{4}\left(\left\|u_{t}\right\|_{2}^{2}+\|u\|_{p+1}^{\frac{2}{1-2 k}}\right), \\
\|u\|_{p+1}^{\frac{2}{1-2 k}}=\|u\|_{p+1}^{p+1}\|u\|_{2}^{\frac{2}{1-2 k}-\frac{1}{p+1}} \leq c_{5} H(0)^{\frac{2}{(1-2 k)(p+1)}-1}\|u\|_{p+1}^{p+1} .
\end{gathered}
$$

Then 4.63) takes the form

$$
L(t)^{\varpi} \leq c_{6}\left(H(t)+\left\|u_{t}\right\|_{2}^{2}+\|u\|_{p+1}^{p+1}\right) .
$$

Combining (4.63) and (4.66) we deduce that

$$
L^{\prime}(t) \geq c_{7} L(t)^{\varpi}, c_{7}>0, \varpi>1
$$

A simple integration over $(0, t)$ yields the desired result.

\section{References}

[1] V. Georgiev, D. Todorova, Existence of solutions of the wave equations with nonlinear damping and source terms, J. Differential Equations. 109 (1994) 295-308.

[2] H.A. Levine, Some additional remarks on the nonexistence of global solutions to nonlinear wave equations, SIAM J. Math. Anal. 5 (1974) 138-146.

[3] H.A. Levine, S. Ro Park, Global existence and global nonexistence of solutions of the Cauchy problems for a nonlinearly damped wave equation, J. Math. Anal. Appl. 228 (1998) 181-205.

[4] K. Ono, Global existence, decay and blow up of solutions for some mildly degenerate nonlinear Kirchhoff string, J. Differential Equations. 137 (1997) 273-301.

[5] Xiaosen Han, Mingxin Wang, global existence and blow up of solutions for a system of nonlinear viscoelastic wave equations with damping and source, Nonlinear Analysis. 71 (2009) 5427-5450.

[6] Shun-Tang Wu, On decay and blow up of solutions for a system of nonlinear wave equations, J. Math. Anal. Appl. 394 (2012) 360-377.

[7] M. Nakao, Existence and Decay of Global Solutions of Some Nonlinear Degenerate Parabolic Equation, Journal of Mathematical Analisis and Applications. 109(1985) 118-129.

[8] M. Nakao, Y. Ohara, Gradient Estimates of Periodic Solutions for Some Quasilinear Parabolic Equations, Journal of Mathematical Analisis and Applications. 204(1996) 868-883.

[9] M. Gobbino, Quasilinear Degenerate Parabolic Equations of Kirchhof Type, Mathematical Methods in Applied Sciences. 22 (1999) 375-388.

[10] S. Berrimi, S. A. Messaoudi, A decay result for a quasilinear parabolic system, Progress in Nonlinear Diferential Equations and their Applications. 53 (2005) 43-50.

[11] R. Datko, J. Lagnese, M. P. Polis, An example on the effect of time delays in boundary feedback stabilization of wave equations, SIAMJ.Control Optim. 24(1986) 152-156.

[12] S. Nicaise, C. Pignotti, Stabilization of the wave equation with boundary or internal distributed delay, Differential Integral Equations. 21(2008) 935-958.

[13] S. A. Messaoudi, Blow up of positive-initial-energy solutions of a nonlinear viscoelastic hyperbolic equation, J. Math. Anal. Appl. 320 (2006) 902-915.

[14] M. Nakao, A difference inequality and its application to nonlinear evolution equations, J. Math. Soc. Japan. 30 (1978) 747-762.

[15] K. Ammari, Serge Nicaise, Cristina Pignotti, Feedback boundary stabilization of wave equations with interior delay, Systems and Control Letters. 59(2010) 623-628.

[16] A. Benaissa, K. Zennir, Existence and asymptotic behavior of solutions for a nonlinear wave equation with a delay term, Math. Methods Appl. Sci. 59(2011) 620-645.

[17] M. Aassila, Stability and asymptotic behaviour of solutions of the heat equation, IMAJ. Appl. Math. 69(1)(2004) 93-109.

[18] T. Matsuyama, R. Ikehata, On global solutions and energy decay for the wave equation of Kirchhoff type with nonlinear damping terms, J. Math. Anal. Appl. 204(3) (1996) 729-753.

[19] M. Nakao, Global existence of classical solutions to the initial-boundary value problem of the semilinear wave equations with a degenerate dissipative term, Nonlinear Anal. 15(2) (1990) 115-140. 
[20] M. Nakao, Existence of global smooth solutions to the initial-boundary value problem for the quasi-linear wave equation with a degenerate dissipative term, J. Differential Equations. 98(2) (1992) 299-327.

[21] K. Ono, Blowing up and global existence of solutions for some degenerate nonlinear wave equations with some dissipation, Nonlinear Anal. 30(7) (1997) 4449-4457.

[22] K. Ono, Global existence, decay and blow-up of solutions for some mildly degenerate nonlinear Kirchhoff strings, J. Differential Equations. 137(2) (1997) 273-301.

[23] Shun-Tang Wu, L.-Y. Tsai, On global existence and blow-up of solutions for an integro-differential equation with strong damping, Taiwanese J. Math. 10 (4)(2006) 979-1014.

[24] R. Zeng, C. L. Mu, S. M. Zhou, A blow-up result for Kirchhoff-type equations with high energy, Math. Methods Appl. Sci. 34 (4)(2011) 479-486.

[25] Shun-Tang Wu, Exponential energy decay of solutions for an integro-differential equation with strong damping, J. Math. Anal. Appl. 364 (2)(2010) 609-617.

[26] Shun-Tang Wu, Asymptotic behavior for a viscolastic wave equation with a delay term, J. Taiwanese J. Math. 364 (2)(2013) 765-784.

[27] G. Kirchhof, Vorlesongen iiber Mechanik, Leipzig, Teubner. (1883).

[28] E. V. Stepanova, Decay of the solutions of parabolic equations with double nonlinearity and the degenerate absorption potential, Ukranian Math. J. 364 (4)(2013) 99-121.

[29] S.M. Egnorov, E. Ya, Khruslov, Global weak solutions of the Navier-Stokes-Fokker-Planck system, Ukranian Math. J.364 (4)(2013) 212-248.

[30] J. Liu, Global weak solutions for the weakly dissipative $\mu$-Hunter-Saxton equation, Ukranian Math. J. 364 (4)(2013) 1217-1230.

[31] D. Ouchenane, Kh. Zennir, M. Buyoud, Global weak solutions for the weakly dissipative $\mu$-Hunter-Saxton equation, Ukranian. Math. J.364 (4)(2013) 723-739. 